\title{
The Feasibility of a Modified Exclusive Endoscopic Transcanal Transpromontorial Approach for Vestibular Schwannomas
}

\author{
In Seok Moon ${ }^{1}$ Dongchul Cha ${ }^{1}$ Sung-II Nam ${ }^{2}$ \\ ${ }^{1}$ Department of Otorhinolaryngology, Yonsei University College of \\ Medicine, Seoul, Korea \\ ${ }^{2}$ Department of Otorhinolaryngology, Keimyung University College \\ of Medicine, Daegu, Korea \\ ${ }^{3}$ Department of Otorhinolaryngology, Gyeongsang National \\ University Changwon Hospital, Changwon, Korea
}

J Neurol Surg B 2019;80:82-87.

\begin{abstract}
Keywords

- endoscopy

- ear canal

- cochlea

- acoustic neuroma

Objective We evaluated the feasibility of an exclusive endoscopic transcanal transpromontorial approach (EETTA) for the treatment of small vestibular schwannomas (VSs) limited to the internal auditory canal (IAC), and introduced a modification without external auditory canal closure.

Methods Between June 2016 and June 2017, seven patients with VS underwent surgery using a modified EETTA. Treatment outcomes, including efficacy of tumor resection, preservation of function, operation time, and quality of life (QOL), were evaluated.

Results The patients preoperatively exhibited Koos Grade I/II tumors and severe-toprofound hearing loss. Gross total resection was accomplished in all cases. There were no major complications, and all patients exhibited normal facial nerve function immediately after surgery. The mean follow-up period was 12.9 months. The operation time (average $196.3 \pm 64.9$ minutes) and hospitalization period (average $7.4 \pm 1.0$ days) were favorable. Short Form-36 scores for QOL showed unremarkable results compared with previous reports.

Conclusions The modified EETTA was effective in the removal of VSs in the IAC. It can be an alternative surgical option for small VSs.
\end{abstract}

\section{Introduction}

The optimal treatment for vestibular schwannomas (VSs) localized in the internal auditory canal (IAC), considering the size and growth rate of the tumor, age, general condition, and hearing status of the patient, remains controversial. ${ }^{1-4}$ Once the decision regarding surgical resection of the tumor is made, the most important factor in determining the surgical approach is the residual hearing of the patient, followed by surgical anatomy and the extent of the tumor. ${ }^{3,5,6}$

received

January 27, 2018

accepted after revision

June 6, 2018

published online

July 30, 2018
DOI https://doi.org/ 10.1055/s-0038-1667061. ISSN 2193-6331.
In patients with serviceable hearing, strategies for the preservation of hearing, such as surgery through the middle cranial fossa and/or retrosigmoid approaches, should be used. In patients with severe-to-profound hearing loss, the translabyrinthine approach (TLA), which provides a wide surgical field and better protection of the facial nerve, is generally used. ${ }^{7,8}$ However, when the tumor is localized to the IAC, a TLA to the IAC involves a long operation time and extensive bony drilling relative to the tumor size. Recently, Marchioni et $\mathrm{al}^{9}$ reported a direct approach to the IAC
License terms

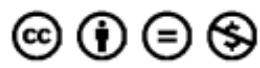

(c) 2019 Georg Thieme Verlag KG Stuttgart · New York
Address for correspondence Jae Young Choi, MD, PhD, Departm
of Otorhinolaryngology, Yonsei University College of Medicine,

(e-msei-ro 50, Seodaemun-gu, 
through the promontory using only an endoscope, referred to as an exclusive endoscopic transcanal transpromontorial approach (EETTA). This confers several advantages over conventional approaches. The EETTA procedure is based on an endoscopic approach, an external incision line is minimal, bony drilling is less, and the risk of blind spots can be minimized.

Here, we report the successful removal of VSs localized in the IAC through a slightly modified EETTA that does not require either closure of the external auditory canal (EAC) or an abdominal incision for fat harvest.

\section{Materials and Methods}

Between June 2016 and June 2017, seven patients underwent surgery through a modified EETTA for VS confined to the IAC at the Department of Otorhinolaryngology, Yonsei University College of Medicine, Seoul, Korea (-Table 1).

The indications for surgery were as follows: (1) the presence of a growing mass or intractable symptom consistent with VS, (2) Koos Grade I or II tumors, ${ }^{10}$ and (3) a hearing status of American Academy of Otolaryngology-Head and Neck Surgery (AAO-HNS) class D (severe-to-profound hearing loss).

All surgeries were performed by a single experienced otological surgeon (I. S. M.). The efficacy of tumor resection, preservation of function, operation time, and quality of life (QOL) were evaluated. ${ }^{11,12}$

\section{Surgical Procedure of Modified EETTA}

The term exclusive was used to indicate that all procedures were performed using only an endoscope, without the assistance of a microscope. The surgical steps were similar to those described by Marchioni et al (-Fig. 1), ${ }^{9,13,14}$ except for the following modifications: (1) tracing of the tympanic segment and geniculate ganglion of the facial nerve first instead of mastoid segment exposure, (2) use of microdrills instead of a piezosurgery instrument, (3) no closure of the EAC, (4) cartilage myringoplasty, and (5) no incision of the abdomen.

The following equipment was prepared for surgery through the modified EETTA: 0-degree, 30-degree, and 3-mm endoscopes (Storz, Tuttlingen, Germany); Panetti set for endoscopic ear surgery (Spiggle \& Theis, Overath, Germany); special probes connecting to NIM 2.0 nerve monitoring system (Medtronic, Minneapolis, MN); and a powered, curved sharp-tipped micro drill system (Sichuan, China).

The patient was placed in a supine position, with the head rotated to the contralateral side. The endoscope was inserted into the EAC in a manner similar to that used in "traditional" endoscopic ear surgery. Then, a circumferential incision was made on the skin of the EAC (7-10 $\mathrm{mm}$ from the tympanic annulus). The tympanic membrane and the skin of the EAC were elevated and harvested en bloc, exposing the tympanic cavity (-Fig. 2A). Circumferential drilling of the EAC was performed to increase the view further and to facilitate maneuvering of the surgical instruments into the canal. The incudostapedial joint was separated, and the incus was removed. The tensor tympani tendon was excised and the malleus was removed to allow wide exposure of the entire tympanic tract of the facial nerve (-Fig. 2B). The tensor muscle was then drilled using a microdiamond burr until the geniculate ganglion was visible to identify the facial nerve tympanic segment located between the cochleariform process and the cog. To approach the IAC through an EETTA, it is necessary to drill the cochlea. However, because the tympanic segment and geniculate ganglion are located in the superior portion of the cochlea, it is safer to start with promontory drilling after identification of this area.

Marchioni et $\mathrm{al}^{9,13,14}$ recommended exposure of the mastoid segment of the facial nerve by extensive drilling of the posterior wall and setting of the exposed facial nerve as the posterior margin of the surgical field. However, in the modified EETTA, because tumor removal was followed by myringoplasty with harvesting of the cartilage, the posterior wall should be preserved where possible. The jugular bulb was visualized in the tympanic cavity by drilling the hypotympanic area, which represents the inferior limit of this approach. The stapes was removed from the oval window to expose the vestibule (-Fig. $\mathbf{2 C}$ ). The inferior lip of the oval window was gently removed using a microdrill to enlarge the opening and expose the saccular fossa in the medial aspect of the vestibule (-Fig. 2D). The promontory was then drilled to expose the basal, middle, and apical turns of the cochlea (-Fig. 2E, F). Photographs of the surgery are available as a supplementary file (-Supplementary Fig. 1 and - Video $\mathbf{1}$ ).

Table 1 Detailed characteristics of the patients

\begin{tabular}{|l|l|l|l|l|l|l|l|l|l|l|}
\hline $\begin{array}{l}\text { Patient } \\
\text { no. }\end{array}$ & Sex & Age & Site & Disease & $\begin{array}{l}\text { Follow-up, } \\
\text { months }\end{array}$ & $\begin{array}{l}\text { CPA } \\
\text { extension, } \\
\text { mm }\end{array}$ & $\begin{array}{l}\text { Tumor } \\
\text { control }\end{array}$ & Complication & $\begin{array}{l}\text { Operation } \\
\text { time, } \\
\text { minutes }\end{array}$ & $\begin{array}{l}\text { Hospital } \\
\text { stay, days }\end{array}$ \\
\hline 1 & Female & 59 & Left & VS & 18 & 5 & Total & None & 245 & 9 \\
\hline 2 & Female & 58 & Right & VS & 18 & 4 & Total & None & 318 & 8 \\
\hline 3 & Female & 51 & Right & CS & 17 & 0 & Total & None & 167 & 7 \\
\hline 4 & Female & 55 & Left & VS & 15 & 0 & Total & None & 149 & 7 \\
\hline 5 & Male & 58 & Right & VS & 8 & 0 & Total & None & 128 & 8 \\
\hline 6 & Male & 64 & Right & VS & 8 & 0 & Total & None & 157 & 7 \\
\hline 7 & Male & 42 & Left & VS & 6 & 0 & Total & None & 210 & 6 \\
\hline
\end{tabular}

Abbreviations: CPA, cerebellar pontine angle; CS, cochlear schwannoma; VS, vestibular schwannoma. 
A

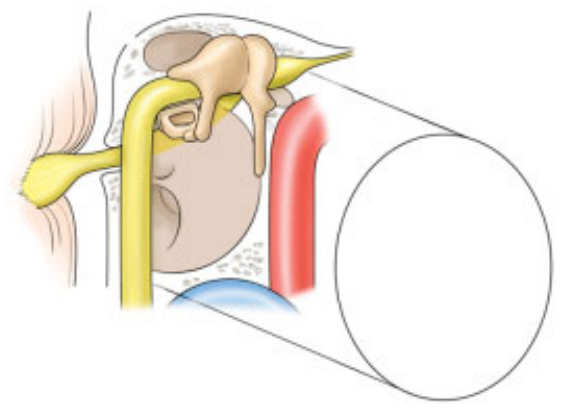

C

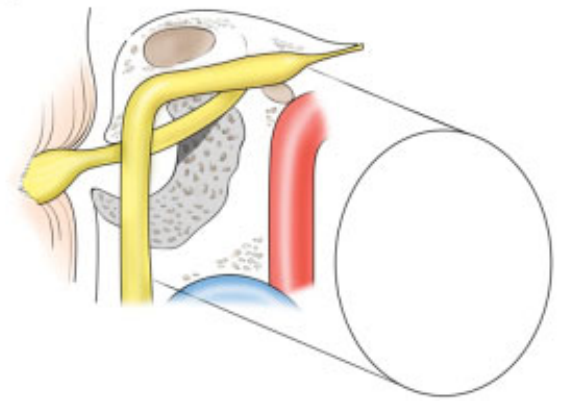

B

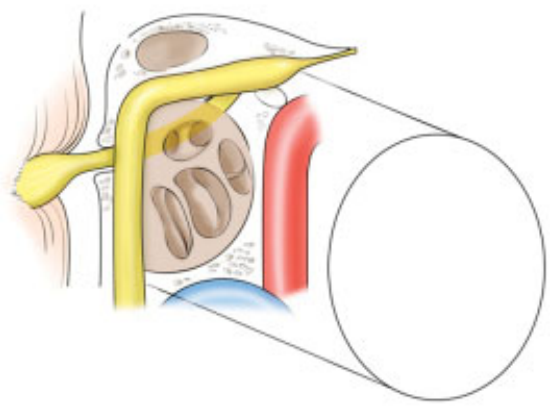

D

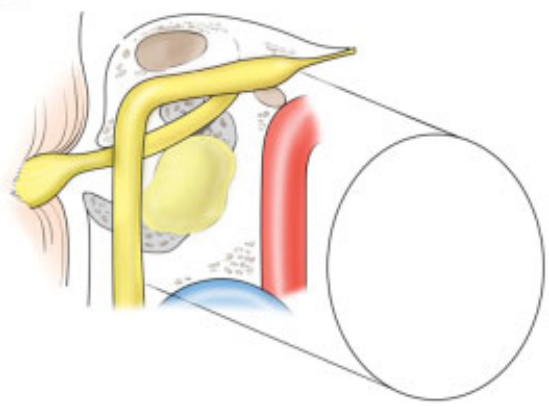

Fig. 1 Schematic representation of the modified exclusive endoscopic transcanal transpromontorial approach (EETTA). (A) An endoscope was inserted into the external auditory canal (EAC) in a similar manner to that in conventional endoscopic ear surgery. The tympanic membrane and the skin of the EAC were elevated and harvested en bloc, exposing the tympanic cavity. (B) The tympanic segment and geniculate ganglion of the facial nerve were traced, and the promontory was drilled to expose the internal auditory canal (IAC). (C) After complete drilling of the cochlea, the IAC was exposed and anatomical limitations (anteriorly carotid artery, inferiorly jugular bulb, superiorly facial nerve) were identified. (D) The vestibular schwannoma was exposed and endoscopically removed through the transpromontorial tract.

\section{Video 1}

Surgical video of modified exclusive endoscopic transcanal transpromontorial approach. Online content including video sequences viewable at: www. thieme-connect.com/products/ejournals/html/ 10.1055/s-0038-1667061.

Upon exposure of the modiolus, which is the lateral end of the IAC and appears as a roof or cover in the surgical view, caution should be exercised to avoid damaging the structure during the subsequent procedure. Unintended damage of the roof causes cerebrospinal fluid (CSF) leakage, which hinders subsequent drilling, as well as exposure of the dura and/or the tumor, which would result in a longer and more challenging surgical procedure.

At this stage of the procedure, the labyrinthine tract of the facial nerve passes through an imaginary line behind the geniculate ganglion to the spherical recess, just above the apical turn of the cochlea. Next, drilling was performed around the inferior aspect of the vestibule until the fundus and the middle portion of the IAC were identified, exposing the lateral most aspect of the pathology. The IAC was exposed extensively by further drilling around the inferior aspect of the vestibule.
The superior, anterior, and inferior limits of dissection were the tympanic tract of the facial nerve, internal carotid artery, and jugular bulb. Circumferential drilling was continued under the vestibule and around the IAC until the reflection of the dura of the IAC meatus was uncovered ( - Fig. 2G). An incision of the dura was made along the IAC to expose the tumor in the canal. When the mass occupied the entire IAC, central debulking was performed to improve management of the tumor, allowing CSF drainage from the cerebellar pontine angle (CPA) and facilitating visualization of the facial nerve in the IAC. Next, the tumor was gently dissected from the facial nerve inside the IAC. The VS was removed from the meatus to the fundus of the IAC, preserving the facial nerve (-Fig. $\mathbf{2 H}$ ). A nerve-monitoring device for facial nerve is essential for this step. The tumor can be enucleated if it is very small and should be removed piecemeal until total removal is achieved ( - Fig. 21). Following tumor resection, the surgical field was reexamined using a 30degree endoscope to identify any possible residual disease. The IAC segment of the facial nerve was then traced (-Fig. 2 J).

The tragal cartilage, perichondrium, soft tissue in the tragal area, and ear lobule fat were harvested. Ear lobule fat and soft tissue from the tragal area were plugged with SURGICEL (Ethicon Inc., Cincinnati, $\mathrm{OH}$ ) into the inner ear entrance to prevent CSF leakage ( $\mathbf{- F i g}$. $\mathbf{2 K}$ ). A cartilage tympanoplasty was performed (-Fig. 2L). Elective lumbar drainage was performed in three patients when there was suspicion about the tight plugging of the inner ear entrance. The skin of the EAC was not closed. 
A

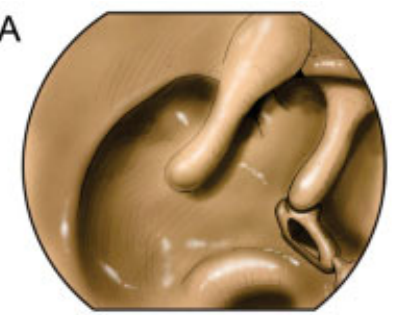

D

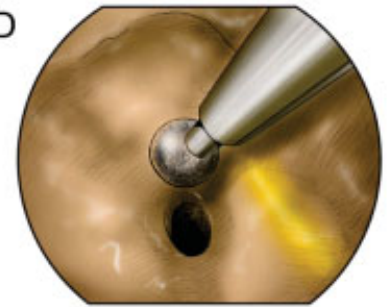

G

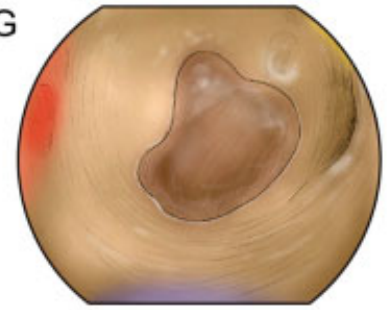

J

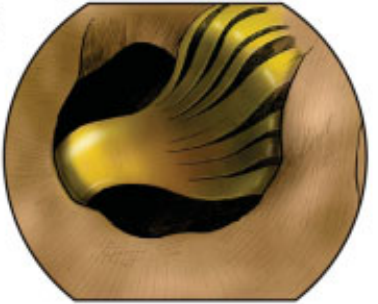

B

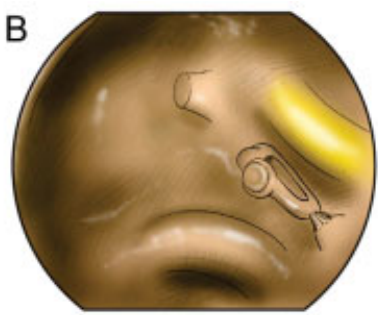

E

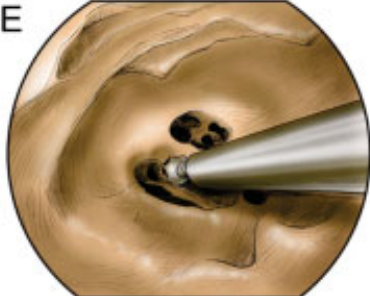

$\mathrm{H}$

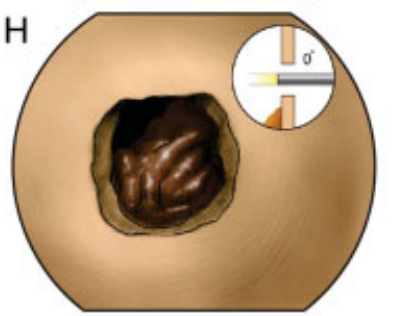

$\mathrm{K}$

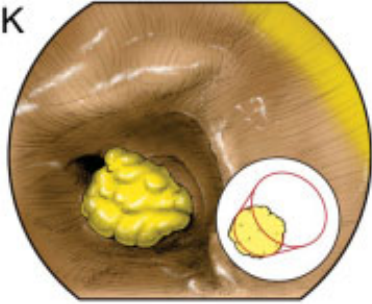

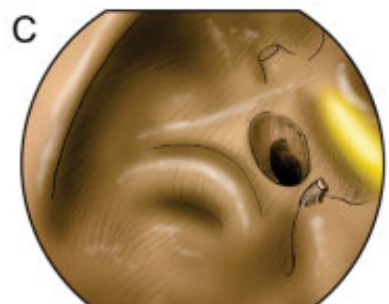

$\mathrm{F}$

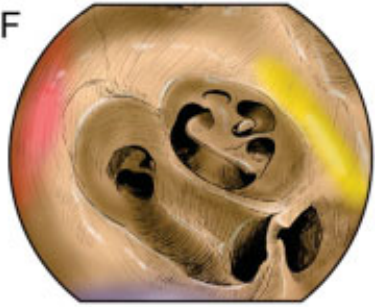

I

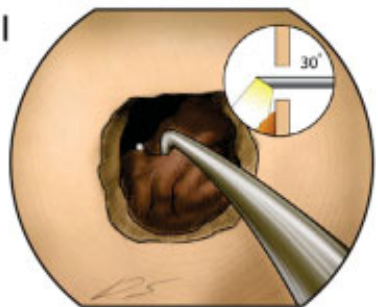

$\mathrm{L}$

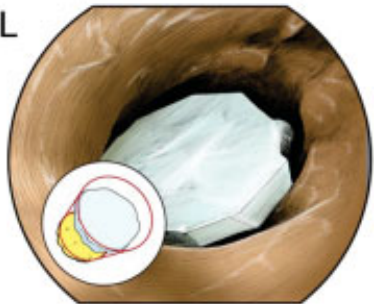

Fig. 2 Surgical procedure. (A) The tympanic membrane and skin of the external auditory canal were elevated and harvested en bloc, exposing the tympanic cavity. (B) The incudostapedial joint was separated, and the incus was removed. The tensor tympani tendon was excised, and the malleus was removed to expose the entire tympanic tract of the facial nerve. The tensor muscle was drilled using a microdiamond burr until the geniculate ganglion was visualized to identify the tympanic segment of the facial nerve. (C) The stapes was removed from the oval window to expose the vestibule. (D) The inferior lip of the oval window was gently removed using microdrills to enlarge the opening and expose the saccular fossa in the medial aspect of the vestibule. (E, F) The promontory was then drilled to expose the basal, middle, and apical turns of the cochlea. (G) Drilling was performed around the inferior aspect of the vestibule until the fundus of the internal auditory canal (IAC) was exposed. $(\mathrm{H})$ The dura of the IAC was exposed completely. The vestibular schwannoma was removed from the meatus to the fundus of the IAC, preserving the facial nerve. The tumor was dissected piecemeal until radical resection was achieved. (I, J) The surgical field was reexamined using a 30-degree endoscope to identify any possible residual disease. The facial nerve was traced and its function was evaluated using a monitoring device. (K) Pieces of the ear lobule fat, tragal perichondrium, and soft tissue were harvested and used to plug the inner ear entrance. (L) The tragal cartilage was harvested, and a cartilage tympanoplasty was performed.

\section{Evaluation of Outcome}

The primary clinical end points were complete tumor resection, verified by immediate postoperative magnetic resonance imaging (MRI) (surgical success), wound healing, and the number of postoperative complications at the final follow-up (functional success).

The secondary end points were operation time, hospitalization period, and perioperative QOL. Operation time was recorded from the start to the end of surgery, hospitalization was recorded from admission (1 day before operation) to discharge, and QOL was subjectively assessed at 1 month after surgery.

\section{Evaluation of Quality of Life}

Perioperative QOL was assessed using the Short Form (SF)-36 questionnaire. This is a generic indicator of health status measured according to eight aspects of health and rated on a scale of 0 to 100 , with higher scores indicating a betterperceived QOL. ${ }^{11,12}$

\section{Results}

This study included seven patients (three males, four females; median age $=55$ years). The mean follow-up period was 12.9 months (-Table $\mathbf{1}$ ).

\section{Efficacy of Tumor Resection}

Gross total resection of the VS and anatomical preservation of the facial nerve were achieved in all patients. As expected, all patients exhibited postoperative deafness on the surgical side as a result of cochlea removal. Histological findings revealed schwannoma in all cases. 


\section{Postoperative Complications}

There were no intraoperative complications. All patients were extubated immediately after surgery. Postoperative computed tomography (CT) performed within 2 hours of surgery revealed no complications. All patients were confined to bed rest and immobilization in the supine position for 48 hours after surgery. There was no instance of CSF leakage, although three patients underwent elective lumbar drainage. Usually, on postoperative day 3, an MRI was taken. All patients exhibited normal facial nerve function immediately after surgery (rough grading system grade I). The tympanic membrane had healed well after 1 month in all patients (-Supplementary Fig. 2 ).

No pressure dressing was applied during the postoperative period; instead, only a light dressing was applied to cover the auricle, and no abdominal dressing was required. The mean operation time was $196.3 \pm 64.9$ minutes, and the average duration of hospitalization was $7.4 \pm 1$ days.

\section{Quality of Life}

Although the SF-36 score does not have a cutoff value, lower scores indicate poorer QOL. SF-36 is categorized as 8 domains and the subscale scores of all eight domain showed scores $>50$ points. Particularly noteworthy were the domains of physical functioning (81.0), bodily pain (89.3), and social functioning (86.5), with scores $>80$ points. Other five domains showed scores of 63 in role limitation due to physical health, 52.8 in general health, 50.8 in energy/fatigue, 76.0 in role limitation due to emotional problems, and 59.3 in mental health.

\section{Discussion}

The optimal protocol for the management of VS in the IAC remains a matter of debate. The general attitude toward the management of VS in the IAC favors a wait-and-scan strategy for most patients by documenting and evaluating the growth of the mass over time. Then, a specific therapeutic approach can be suggested confidently. ${ }^{15-19}$

However, recent studies have shown excellent results for surgery in terms of patient QOL from a long-term point of view. ${ }^{20,21}$ An efficacy of radiosurgery for VS has also been documented, although prospective studies comparing the efficacy of radiosurgery with that of the wait-and-scan strategy are lacking. Thus, it is impossible to define the proportion of tumor growth arrest attributable to radiosurgery and that attributable to the natural course of the disease, as often occurs.

In young patients, the decision to undergo radiosurgery or to follow the wait-and-scan approach does not exclude the need for an operation in the future, which may carry a higher risk of complications. Thus, surgical removal remains the main treatment strategy for VSs. The IAC is a difficult-to-access anatomical region regardless of the surgical approach., ${ }^{3,22}$ While a retrosigmoid approach requires a craniotomy and drilling of the posterior aspect of the petrous bone to expose the IAC completely, gaining access through the TLA requires time-consuming and extensive bone drilling for the identification and proper skeletonization of the IAC. A middle cranial fossa approach involves less bone drilling to expose the petrous bone as compared with a retrosigmoid approach, but it requires a wide craniotomy window as well as temporal lobe retraction. ${ }^{23,24}$ Regardless of the approach chosen, VS surgery is a delicate procedure, with a risk of morbidity, including intra- and postoperative complications. ${ }^{25,26}$

In this study, we describe the feasibility of EETTA and its modification for the treatment of VS. This method offers enables visualization of every structure inside and outside the IAC, including the facial nerve, at high magnification. Moreover, because the procedure is based on an endoscopic approach, an incision line is unnecessary, and the risk of blind spots can be minimized using various angles of the endoscope. The entire course of the facial nerve can be followed using the endoscope, and the modified EETTA guarantees complete removal of the pathology, with very low morbidity of facial function. Moreover, this procedure requires less bony drilling.

Unlike the original procedure, tragal cartilage, perichondrium, soft tissue, and fat in the earlobe are used to plug the inner ear entrance without a closure of the ear canal so that no abdominal incision is necessary and the physiological anatomy can be maintained. Maintenance of the normal anatomy is one of the most important advantages of endoscopic surgery. Our findings demonstrate that the cosmetically excellent outcome achieved with the modified EETTA is comparable to that of tympanoplasty rather than with that of CPA surgery. However, the need for the ear canal in the absence of residual hearing is debatable.

The sample size in this study was small because most patients with an IAC-confined tumor do not require surgical removal of the tumor, and patients for surgery should thus be selected carefully. Based on our experience with seven cases, we suggest the following indications for the modified EETTA in patients with VS: (1) a growing Koos Grade I VS or cochlear schwannoma, (2) an AAO-HNS class D hearing status, and (3) symptoms unresponsive to medical treatment. Despite these limited criteria in current time, the modified EETTA is a promising technique because of its advantages over conventional microscopic approaches in terms of mobility and cosmetically excellence outcomes. The positive outcomes observed in this study are possibly attributable to the early stage of the VS as well as to the loose adhesion of the mass to the nerve. It has to be noted, however, that preservation of hearing using this approach is not feasible.

\section{Conclusions}

Surgery through a modified EETTA is a shortcut to VSs, especially in the IAC, and can minimize external incision and internal blind spots. Thus, the modified EETTA described here can be an excellent treatment option for tumors localized in the IAC.

\section{Funding}

This study was supported by a grant from the National Research Foundation of Korea (NRF) funded by the Ministry of Education (2014R1A1A2058141). 


\section{Conflicts of Interest}

The authors have no conflicts of interest to declare.

\section{Acknowledgment}

The authors would like to thank Dong-Su Jang, MFA (Medical Illustrator, Medical Research Support Section, Yonsei University College of Medicine, Seoul, Korea) for his help with the illustrations.

\section{References}

1 Kennedy RJ, Shelton C, Salzman KL, Davidson HC, Harnsberger HR. Intralabyrinthine schwannomas: diagnosis, management, and a new classification system. Otol Neurotol 2004;25(02):160-167

2 Meyer TA, Canty PA, Wilkinson EP, Hansen MR, Rubinstein JT, Gantz BJ. Small acoustic neuromas: surgical outcomes versus observation or radiation. Otol Neurotol 2006;27(03):380-392

3 Neff BA, Willcox TO Jr, Sataloff RT. Intralabyrinthine schwannomas. Otol Neurotol 2003;24(02):299-307

4 ThakurJD, Banerjee AD, Khan IS, et al. An update on unilateral sporadic small vestibular schwannoma. Neurosurg Focus 2012;33(03):E1

5 Falcioni M, Taibah A, Di Trapani G, Khrais T, Sanna M. Inner ear extension of vestibular schwannomas. Laryngoscope 2003;113 (09):1605-1608

6 Mohr G, Sade B, Dufour J-J, Rappaport JM. Preservation of hearing in patients undergoing microsurgery for vestibular schwannoma: degree of meatal filling. J Neurosurg 2005;102(01):1-5

7 Arriaga MA, Lin J. Translabyrinthine approach: indications, techniques, and results. Otolaryngol Clin North Am 2012;45(02):399-415, ix

8 Irving RM, Jackler RK, Pitts LH. Hearing preservation in patients undergoing vestibular schwannoma surgery: comparison of middle fossa and retrosigmoid approaches. J Neurosurg 1998;88(05): 840-845

9 Marchioni D, Alicandri-Ciufelli M, Rubini A, Masotto B, Pavesi G, Presutti L. Exclusive endoscopic transcanal transpromontorial approach: a new perspective for internal auditory canal vestibular schwannoma treatment. J Neurosurg 2017;126(01):98-105

10 Koos W, Speltzer Reds. Microsurgery of cerebellopontine angle tumors In: Clinical Microneurosurgery. Stuttgart: Thieme; 1976

11 da Cruz MJ, Moffat DA, Hardy DG. Postoperative quality of life in vestibular schwannoma patients measured by the SF36 Health Questionnaire. Laryngoscope 2000;110(01):151-155

12 Myrseth E, Møller P, Pedersen P-H, Lund-Johansen M. Vestibular schwannoma: surgery or gamma knife radiosurgery? A prospective, nonrandomized study. Neurosurgery 2009;64(04):654-661, discussion 661-663
13 Marchioni D, Carner M, Rubini A, et al. The fully endoscopic acoustic neuroma surgery. Otolaryngol Clin North Am 2016;49 (05):1227-1236

14 Presutti L, Alicandri-Ciufelli M, Cigarini E, Marchioni D. Cochlear schwannoma removed through the external auditory canal by a transcanal exclusive endoscopic technique. Laryngoscope 2013; 123(11):2862-2867

15 Bennett M, Haynes DS. Surgical approaches and complications in the removal of vestibular schwannomas. Otolaryngol Clin North Am 2007;40(03):589-609, ix-X

16 Charabi S, Thomsen J, Mantoni M, et al. Acoustic neuroma (vestibular schwannoma): growth and surgical and nonsurgical consequences of the wait-and-see policy. Otolaryngol Head Neck Surg 1995;113(01):5-14

17 Di Lella F, Dispenza F, De Stefano A, Falcioni M, Sanna M. Management of intralabyrinthine schwannomas. Auris Nasus Larynx 2007;34(04):459-463

18 Perez R, Chen JM, Nedzelski JM. Intratemporal facial nerve schwannoma: a management dilemma. Otol Neurotol 2005;26 (01):121-126

19 Stangerup S-E, Thomsen J, Tos M, Cayé-Thomasen P. Long-term hearing preservation in vestibular schwannoma. Otol Neurotol 2010;31(02):271-275

20 Gosselin É, Maniakas A, Saliba I. Meta-analysis on the clinical outcomes in patients with intralabyrinthine schwannomas: conservative management vs. microsurgery. Eur Arch Otorhinolaryngol 2016;273(06):1357-1367

21 Kim HJ, Jin Roh K, Oh HS, Chang WS, Moon IS. Quality of life in patients with vestibular schwannomas according to management strategy. Otol Neurotol 2015;36(10):1725-1729

22 Miller RS, Pensak ML. An anatomic and radiologic evaluation of access to the lateral internal auditory canal via the retrosigmoid approach and description of an internal labyrinthectomy. Otol Neurotol 2006;27(05):697-704

23 Colletti V, Fiorino F. Is the middle fossa approach the treatment of choice for intracanalicular vestibular schwannoma? Otolaryngol Head Neck Surg 2005;132(03):459-466

24 Colletti V, Fiorino F. Middle fossa versus retrosigmoid-transmeatal approach in vestibular schwannoma surgery: a prospective study. Otol Neurotol 2003;24(06):927-934

25 Samii M, Matthies C. Management of 1000 vestibular schwannomas (acoustic neuromas): surgical management and results with an emphasis on complications and how to avoid them. Neurosurgery 1997;40(01):11-21, discussion 21-23

26 Sanna M, Taibah A, Russo A, Falcioni M, Agarwal M. Perioperative complications in acoustic neuroma (vestibular schwannoma) surgery. Otol Neurotol 2004;25(03):379-386 